\title{
A Stabilizing Controller for Regulation of UAV With Manipulator
}

\author{
Min Jun Kim ${ }^{(1)}$, Konstantin Kondak, and Christian Ott ${ }^{(1)}$
}

\begin{abstract}
Stability is not trivial in aerial manipulation tasks because of the dynamical coupling between the aerial vehicle and the manipulator. This is especially true when the manipulator becomes heavy, so that its dynamics can be significant. In this letter, a stabilizing controller for the regulation of overall system will be designed without using any assumptions on the dynamic model. In addition, thorough discussion with simulation validation is presented.
\end{abstract}

Index Terms-Aerial systems: mechanics and control, motion control.

\section{INTRODUCTION}

I $\mathrm{N}$ RECENT years, as a natural extension of successful achievements in unmanned aerial vehicle (UAV) studies, aerial manipulation has become an emerging research field. In this field, there are many interesting research branches such as mechanical design [1]-[4], modeling methodology [5], [6], and intelligence [7]-[9]. In addition to these, control design is also an important branch to accomplish successful aerial manipulation.

Control of UAV-manipulator (in short, UAV-M hereinafter) system is a quite complicated problem because the UAV is dynamically coupled with the robotic manipulator, and moreover, the UAV is typically under-actuated. ${ }^{1}$ Control problem becomes especially important when the dynamics of the manipulator becomes significant. One example is the UAV-M in Fig. 1(a) which is equipped with a 7 Degrees of Freedom (DoF) manipulator. Since the manipulator weighs about $14 \mathrm{~kg}$ (while the UAV body weighs around $35 \mathrm{~kg}$ ), the dynamics of the manipulator can significantly affect that of the UAV, and vice versa. Our previous study [11] has shown that, while the UAV is hovering, manipulator's simple swing motion can actually cause instability. To overcome this, [12] showed that UAV and manipulator can be controlled independently if the Center of Mass (CoM) of the whole system lies on the gravity vector of the UAV. For example, [8], [13] used this strategy in the aerial manipulation tasks. However, the analysis was rather conceptual because it was based on a simplified model, and formal stability proof was not provided.

Although several approaches have employed full dynamics of the UAV-M systems in control design [5], [14]-[20], formal

Manuscript received September 10, 2017; accepted January 13, 2018. Date of publication February 7, 2018; date of current version February 27, 2018. This letter was recommended for publication by Associate Editor V. Lippiello and Editor J. Roberts upon evaluation of the reviewers' comments. This work was supported by the funding of the European Commission to the AEROARMS project under the H2020 Programme (Grant Agreement 644271). This paper is part of IEEE Robotics and Automation Letters' Special Issue on Aerial Manipulation, edited by F. Ruggiero, V. Lippiello, and A. Ollero. (Corresponding author: Min Jun Kim.)

The authors are with the Institute of Robotics and Mechatronics, German Aerospace Center (DLR), Wessling 82234, Germany (e-mail: minjun. kim@dlr.de; konstantin.kondak@dlr.de; christian.ott@dlr.de).

Digital Object Identifier 10.1109/LRA.2018.2803205

${ }^{1}$ As a matter of fact, fully-actuated UAVs are also being studied [10].

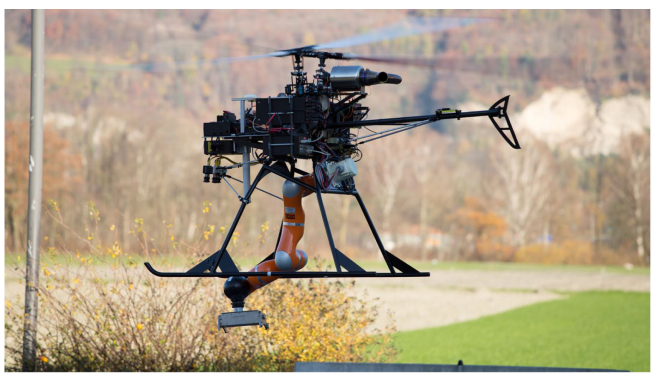

(a)

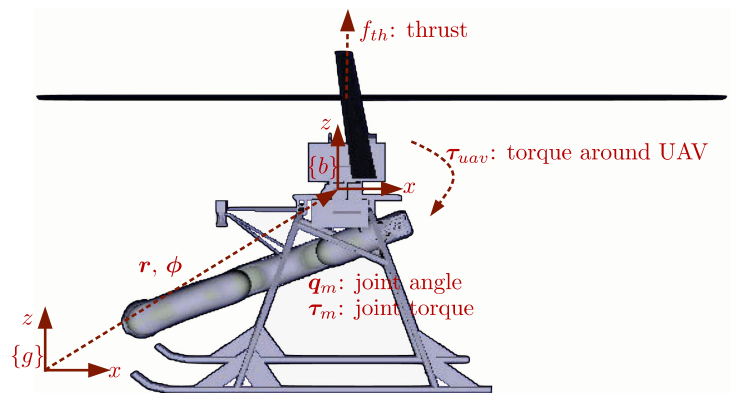

(b)

Fig. 1. (a) UAV-M system developed in DLR. (b) Schematic diagram of the system when the joint angles are zero. This letter assumes that the user can command thrust force $\left(f_{t h}\right)$, torque around the UAV ( $\left.\boldsymbol{\tau}_{\text {uav }}\right)$, and joint torque $\left(\boldsymbol{\tau}_{m}\right)$ to the UAV-M. However, for our system, angular velocity can be commanded instead of $\boldsymbol{\tau}_{\text {uav }}$. This feature will be discussed in Section IV. For any cases, the system does not have actuation along the body $x, y$ directions (under-actuation).

stability proof was often missing or was based on some assumptions of the UAV-M model. [14], [15] analyzed admissible manipulator motion that does not harm flight stability, and [16] solved optimization problem to find a manipulator trajectory that minimizes the coupling dynamics. [5], [17] provided great insight into UAV-M control problems, but the controller was designed only for planar systems. In some studies, the control law was designed as if the UAV-M has full actuation, and is distributed to actuators using least square [18] or small angle assumption [19]. A recent study [20] showed that the UAV-M is a flat system if the manipulator is attached to the CoM of the UAV and is operating in a plane, which can be considered to be almost true for many systems. However, these assumptions seems hard to be verified on our system because the manipulator is mounted far from the CoM and is able to move in 3D.

Therefore, in this letter, we solve a regulation problem of the UAV-M systems without any assumptions on the UAV-M model in the stability proof. Although the regulation is one of the most fundamental questions in control, to the best of authors' 
knowledge, its solution has not been presented yet. The key idea is to express the UAV dynamics in the body frame, so that we have zero force inputs along $x$ and $y$ directions in the equation of motion (recall that UAV is under-actuated; there is no input along body $x, y$ directions as shown in Fig. 1(b)). In contrast, when the UAV dynamics is expressed in the global reference frame, the thrust input influences every $x, y, z$ directions via rotation matrix. Due to the zero inputs, the formulation can be handled easily. A stabilizing controller can be designed by coupling the body linear velocity with body angular velocity properly.

Another advantage of the proposed controller is that it has a clear working principle which (i) assigns physical meanings to the control gains, and (ii) allows us to apply the proposed controller to the UAV-M which has an angular velocity servo instead of a torque servo for the UAV part (for instance, our system in Fig. 1). In addition, the limitation of the proposed controller in the tracking scenario will be also discussed.

The rest of the paper is organized as follows. Section II presents the modeling of the UAV-M, and Section III presents the control design. Discussions on the controller will be presented in Section IV with simulation validation in Section V. Section VI concludes the paper.

\section{MODELING OF UAV-MANIPULATOR SySTEMS}

\section{A. Notations}

The following notations are used throughout the paper.

- $\boldsymbol{q}_{m} \in \Re^{n}:$ Generalized coordinates of the $n$ DoF robotic manipulator.

- $\boldsymbol{v}_{b}=\left[\begin{array}{lll}v_{b, x} & v_{b, y} & v_{b, z}\end{array}\right]^{T}$ (or $\boldsymbol{w}_{b}$ ): Body linear (angular) velocity. The body frame $\{b\}$ is located on the CoM of the UAV.

- $\boldsymbol{\xi}=\left[\begin{array}{llll}\boldsymbol{v}_{b}^{T} & \boldsymbol{w}_{b}^{T} & \dot{\boldsymbol{q}}_{m}^{T}\end{array}\right]^{T}$ : UAV-M velocity vector.

- $\boldsymbol{v}$ : Linear velocity represented in the global frame $\{g\}$.

- $r$ : The origin of $\{b\}$ from that of $\{g\}$, represented in $\{g\}$. Note that $\boldsymbol{v}=\dot{\boldsymbol{r}}$.

- ${ }^{g} \boldsymbol{R}_{b}$ : The orientation of $\{b\}$ frame seen from the $\{g\}$ frame ( ${ }^{b} \boldsymbol{R}_{g}$ is the inverse).

- $\phi=[\alpha \beta \gamma]^{T}$ : Roll, pitch, and yaw angles.

- $Q(\phi)$ : The map from Euler rate to the body angular velocity; namely, $\boldsymbol{w}_{b}=\boldsymbol{Q}(\boldsymbol{\phi}) \dot{\boldsymbol{\phi}}$.

- $f_{t h}, \tau_{\text {uav }}$ : Thrust and torque inputs on the UAV represented in the body frame.

- $\tau_{m}$ : Joint torque input on the manipulator.

- $\boldsymbol{M} \in \Re^{(6+n) \times(6+n)}, \boldsymbol{C} \in \Re^{(6+n) \times(6+n)}$, and $\boldsymbol{g} \in \Re^{6+n}$ : Inertia matrix, Coriolis and centrifugal matrix, and gravity vector represented in $\{b\}$, respectively.

- The first 3 by $n+6$ components of $M$ and $C$ can be expressed as

$$
\begin{aligned}
\left.M_{t} \triangleq M\right|_{\text {first } 3 \times(n+6)} & =\left[\begin{array}{lll}
M_{t t} & M_{t r} & M_{t m}
\end{array}\right], \\
\left.C_{t} \triangleq C\right|_{\text {first } 3 \times(n+6)} & =\left[\begin{array}{lll}
C_{t t} & C_{t r} & C_{t m}
\end{array}\right] .
\end{aligned}
$$

Here, the subscripts $t, r$, and $m$ represent 'translational', 'rotational', and 'manipulator', respectively. Similarly, $\boldsymbol{g}_{t}$ represents the first three elements of $\boldsymbol{g}$.

- $\vec{M}, \bar{C}$, and $\overline{\boldsymbol{g}}$ : Dynamic parameters evaluated at the desired manipulator's configuration $\boldsymbol{q}_{m}=\boldsymbol{q}_{m}^{\text {des }}$. Similar to the previous, $\overline{\boldsymbol{g}}_{t}=\left[\begin{array}{lll}\bar{g}_{t, x} & \bar{g}_{t, y} & \bar{g}_{t, z}\end{array}\right]^{T}$ represents the first three component of $\overline{\boldsymbol{g}}$.

\section{B. Modeling}

The equation of motion of UAV-M in the body frame can be expressed as

$$
\boldsymbol{M}\left(\boldsymbol{q}_{m}\right) \dot{\boldsymbol{\xi}}+\boldsymbol{C}\left(\boldsymbol{q}_{m}, \boldsymbol{\xi}\right) \boldsymbol{\xi}+\boldsymbol{g}\left(\boldsymbol{\phi}, \boldsymbol{q}_{m}\right)=\boldsymbol{\tau}_{b}
$$

with previously introduced notations. Here, $\tau_{b}$ represents the control command in the body frame, and is given by

$$
\boldsymbol{\tau}_{b}=\left[\begin{array}{lllll}
0 & 0 & f_{\text {th }} & \boldsymbol{\tau}_{\mathrm{uav}}^{T} & \boldsymbol{\tau}_{m}^{T}
\end{array}\right]^{T},
$$

where $f_{\text {th }} \in \Re, \boldsymbol{\tau}_{\text {uav }} \in \Re^{3}$, and $\tau_{m} \in \Re^{n}$ are control inputs to be designed. Note that, by expressing the dynamic model in the body frame, the first two elements of $\tau_{b}$ are zero. This makes it easy to handle the equation in Section III.

The following two properties of the UAV-M model will be used later.

Property 1: If the roll and pitch angles ( $\alpha$ and $\beta)$ are zero, then the gravitational forces along body $x, y$ directions are zero (i.e., $\bar{g}_{t, x}=0$ and $\bar{g}_{t, y}=0$ ).

Property 2: $\boldsymbol{M}_{t r}$ is given by $\boldsymbol{M}_{t r}=-m^{b} \boldsymbol{r}_{C o M}^{\vee}$. Here, $m$ is the total mass, $(\cdot)^{\vee}$ is the skew-symmetric operator, and ${ }^{b} \boldsymbol{r}_{C o M}$ is the position of the CoM from the origin of $\{b\}$.

\section{A StABILIZING CONTROLLER}

\section{A. Control Goal}

As one of the most fundamental control problems, this letter tackles the regulation task. Namely, we aim at achieving the followings.

- $\boldsymbol{r} \rightarrow \boldsymbol{r}^{\text {des }}$. The desired position of the UAV is given in the global frame.

- $\alpha \rightarrow 0, \beta \rightarrow 0, \gamma \rightarrow \gamma^{\text {des }}$; or in short, $\phi \rightarrow \phi^{\text {des }}=$ $\left[\begin{array}{lll}0 & 0 & \gamma^{\text {des}}\end{array}\right]^{T}$. Roll and pitch angles should be regulated to zero eventually. Otherwise, the UAV will move.

- $\boldsymbol{q}_{m} \rightarrow \boldsymbol{q}_{m}^{\text {des }}$. The robotic arm moves to its desired configuration.

Moreover, one assumption on the control task is made.

Assumption 1: In the desired configuration, the CoM of the overall system does not lie on the $x-y$ plane of the $\{b\}$ frame. ${ }^{2}$ Note that, under this assumption, the first 2 by 2 components of $M_{t r}$ has full rank from Property 2 .

\section{B. Control Design}

This section proposes a stabilizing controller for the regulation of UAV-M systems. One of the main difficulties in accomplishing the control goal is the under-actuation nature. Note that, due to the absence of control inputs along body $x, y$ directions, it is not possible to control the UAV position along these directions directly. Physically speaking, however, it can be controlled indirectly by controlling the orientation of the UAV. To realize this physical intuition, the proposed approach first applies feedback linearization to an output variable composed of $\boldsymbol{w}_{b}$ and $\dot{\boldsymbol{q}}_{m}$. Then the classical pole-placement technique is applied only to the latter component, which allows us to decouple the manipulator dynamics from the UAV dynamics by employing the cascade control theory. The remaining output $\boldsymbol{w}_{b}$ will be coupled with the UAV position (and velocity) properly to achieve asymptotic stability.

\footnotetext{
${ }^{2}$ This may limit the workspace, but not likely to be restrictive in practice because the workspace of the UAV-M is usually below the UAV.
} 
To begin with, let us rewrite (3) as follows to highlight that the UAV-M is an under-actuated system

$$
\boldsymbol{M} \dot{\boldsymbol{\xi}}+\boldsymbol{C} \boldsymbol{\xi}+\boldsymbol{g}=\boldsymbol{B}_{u} \boldsymbol{u}+\boldsymbol{B}_{t h} f_{t h},
$$

where

$$
\begin{aligned}
\boldsymbol{B}_{u} & =\left[\begin{array}{c}
\mathbf{0}_{3 \times(3+n)} \\
\boldsymbol{I}_{(3+n) \times(3+n)}
\end{array}\right] \in \Re^{(6+n) \times(3+n)}, \\
\boldsymbol{B}_{t h} & =\left[\begin{array}{c}
\mathbf{0}_{2 \times 1} \\
1 \\
\mathbf{0}_{(3+n) \times 1}
\end{array}\right] \in \Re^{(6+n) \times 1} .
\end{aligned}
$$

Here, $f_{\text {th }}$ and $\boldsymbol{u}=\left[\boldsymbol{\tau}_{\text {uav }}^{T} \boldsymbol{\tau}_{m}^{T}\right]^{T}$ are the control inputs to be designed.

From Fig. 1(b), it is natural to select $f_{t h}$ as a dedicated control input for body $z$ direction. Another control input $\boldsymbol{u}$ will be designed to control an output variable $\boldsymbol{y}$. Since $\boldsymbol{u}$ is composed of the torque around $\operatorname{UAV}\left(\boldsymbol{\tau}_{\text {uav }}\right)$ and joint torques $\left(\boldsymbol{\tau}_{m}\right)$, it is natural to define $y$ by ${ }^{3}$

$$
\begin{aligned}
\boldsymbol{y} & =\underbrace{\left[\boldsymbol{0}_{(3+n) \times 3} \boldsymbol{I}_{(3+n) \times(3+n)}\right]}_{\boldsymbol{B}_{u}^{T}} \boldsymbol{\xi} \\
& =\left(\begin{array}{c}
\boldsymbol{w}_{b} \\
\dot{\boldsymbol{q}}_{m}
\end{array}\right) .
\end{aligned}
$$

To apply feedback linearization, take time derivative of $\boldsymbol{y}$ :

$$
\begin{aligned}
\dot{\boldsymbol{y}} & =\boldsymbol{B}_{u}^{T} \dot{\boldsymbol{\xi}} \\
& =\boldsymbol{B}_{u}^{T} \boldsymbol{M}^{-1}\left(-\boldsymbol{C} \boldsymbol{\xi}-\boldsymbol{g}+\boldsymbol{B}_{u} \boldsymbol{u}+\boldsymbol{B}_{t h} f_{t h}\right) .
\end{aligned}
$$

Then, using

$$
\begin{aligned}
\boldsymbol{u}= & \left(\boldsymbol{B}_{u}^{T} \boldsymbol{M}^{-1} \boldsymbol{B}_{u}\right)^{-1} \\
& \times\left(\boldsymbol{B}_{u}^{T} \boldsymbol{M}^{-1}\left(\boldsymbol{C} \boldsymbol{\xi}+\boldsymbol{g}-\boldsymbol{B}_{t h} f_{t h}\right)+\left(\begin{array}{c}
\dot{\boldsymbol{w}}_{b}^{\mathrm{ref}} \\
\ddot{\boldsymbol{q}}_{m}^{\mathrm{ref}}
\end{array}\right)\right),
\end{aligned}
$$

where $\boldsymbol{B}_{u}^{T} \boldsymbol{M}^{-1} \boldsymbol{B}_{u}$ is always invertible, the closed-loop dynamics of (9) becomes ${ }^{4}$

$$
\dot{\boldsymbol{w}}_{b}=\dot{\boldsymbol{w}}_{b}^{\mathrm{ref}} \text { and } \ddot{\boldsymbol{q}}_{m}=\ddot{\boldsymbol{q}}_{m}^{\mathrm{ref}} .
$$

However, note that (11) does not express the UAV translational dynamics which appears as internal dynamics:

$$
\boldsymbol{M}_{t t} \dot{\boldsymbol{v}}_{b}+\boldsymbol{M}_{t r} \dot{\boldsymbol{w}}_{b}^{\mathrm{ref}}+\boldsymbol{M}_{t m} \ddot{\boldsymbol{q}}_{m}^{\mathrm{ref}}+\boldsymbol{C}_{t} \boldsymbol{\xi}+\boldsymbol{g}_{t}=\left(\begin{array}{c}
0 \\
0 \\
f_{t h}
\end{array}\right)
$$

Now, the problem is to design $f_{t h}, \dot{\boldsymbol{w}}_{b}^{\text {ref }}$ and $\ddot{\boldsymbol{q}}_{m}^{\text {ref }}$ that stabilize the closed-loop UAV-M dynamics (11)-(12).

To make analysis simple, the manipulator dynamics (e.g., $\boldsymbol{M}_{t m} \ddot{\boldsymbol{q}}_{m}^{\mathrm{ref}}$ ) can be eliminated from (12) by employing a wellknown result from the cascaded system control literature [21]. 5

\footnotetext{
${ }^{3} \boldsymbol{y}$ can be chosen alternatively. One example is shown in Appendix.

${ }^{4}$ Note that the reference signal differs from the desired signal in the sense that the former one is a part of control law and the latter is the input to the controller. Namely, the reference signal is designer's choice. By defining the reference properly, the regulation to the desired value can be achieved.

${ }^{5}$ Theorem 1 introduces local stability. Stronger versions can be found in [21, Th. 4.2 and Corollary 4.6]. We only introduce Theorem 1 mainly because the Euler angle representation is local expression. In fact, the proposed controller of this letter satisfies the stronger version as well because it satisfies exponential stability.
}

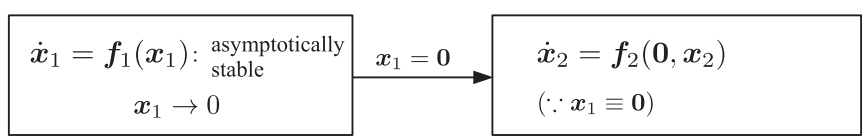

Fig. 2. Cascaded control structure. If $\dot{\boldsymbol{x}}_{1}=\boldsymbol{f}_{1}\left(\boldsymbol{x}_{1}\right)$ is asymptotically stable, then it is sufficient to investigate asymptotic stability of $\dot{\boldsymbol{x}}_{2}=\boldsymbol{f}_{2}\left(\mathbf{0}, \boldsymbol{x}_{2}\right)$ to conclude asymptotic stability of the original system (13)-(14).

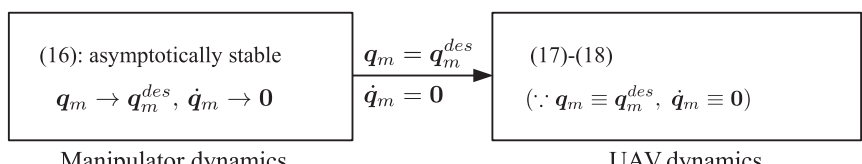

Fig. 3. Similar to Fig. 2, it is sufficient to investigate (17)-(18), because asymptotic (in fact, even exponential) stability of the robotic manipulator can be achieved.

Theorem 1 (Asymptotic stability of cascaded systems): Consider a system

$$
\begin{aligned}
& \dot{\boldsymbol{x}_{1}}=\boldsymbol{f}_{1}\left(\boldsymbol{x}_{1}\right), \\
& \dot{\boldsymbol{x}}_{2}=\boldsymbol{f}_{2}\left(\boldsymbol{x}_{1}, \boldsymbol{x}_{2}\right) .
\end{aligned}
$$

If $\dot{\boldsymbol{x}}_{1}=\boldsymbol{f}_{1}\left(\boldsymbol{x}_{1}\right)$ is locally asymptotically stable to $\boldsymbol{x}_{1}=\mathbf{0}$ and $\dot{\boldsymbol{x}}_{2}=\boldsymbol{f}_{2}\left(\mathbf{0}, \boldsymbol{x}_{2}\right)$ is locally asymptotically stable to $\boldsymbol{x}_{2}=\mathbf{0}$, then (13)-(14) is locally asymptotically stable to $\boldsymbol{x}_{1}=\mathbf{0}$ and $\boldsymbol{x}_{2}=\mathbf{0}$. This result can be described as Fig. 2.

To apply Theorem 1 , let us define

$$
\ddot{\boldsymbol{q}}_{m}^{\mathrm{ref}}=-\boldsymbol{K}_{d} \dot{\boldsymbol{q}}_{m}-\boldsymbol{K}_{p}\left(\boldsymbol{q}_{m}-\boldsymbol{q}_{m}^{\mathrm{des}}\right),
$$

where $\boldsymbol{K}_{d}$ and $\boldsymbol{K}_{p}$ are positive definite gain matrices. The resulting closed-loop dynamics for the manipulator is

$$
\ddot{\boldsymbol{q}}_{m}+\boldsymbol{K}_{d} \dot{\boldsymbol{q}}_{m}+\boldsymbol{K}_{p}\left(\boldsymbol{q}_{m}-\boldsymbol{q}_{m}^{\mathrm{des}}\right)=\mathbf{0}
$$

which falls into the form of (13). The remaining dynamics (i.e., (12) and $\dot{\boldsymbol{w}}_{b}=\dot{\boldsymbol{w}}_{b}^{\text {ref }}$ ) becomes (14).

Hence, according to Theorem 1, it is now sufficient to investigate stability of the following (see Fig. 3):

$$
\begin{aligned}
\overline{\boldsymbol{M}}_{t t} \dot{\boldsymbol{v}}_{b}+\overline{\boldsymbol{C}}_{t t} \boldsymbol{v}_{b}+\overline{\boldsymbol{M}}_{t r} \dot{\boldsymbol{w}}_{b}^{\mathrm{ref}}+\overline{\boldsymbol{C}}_{t r} \boldsymbol{w}_{b}+\overline{\boldsymbol{g}}_{t}=\left(\begin{array}{c}
0 \\
0 \\
f_{t h}
\end{array}\right) \\
\dot{\boldsymbol{w}}_{b}=\dot{\boldsymbol{w}}_{b}^{\mathrm{ref}}
\end{aligned}
$$

Recall that $(\bar{\cdot})$ represents the dynamic parameter $(\cdot)$ evaluated at $\boldsymbol{q}_{m}=\boldsymbol{q}_{m}^{\mathrm{des}}, \dot{\boldsymbol{q}}_{m}=\mathbf{0}$.

The control problem is reduced to design $f_{t h}$ and $\dot{\boldsymbol{w}}_{b}^{\text {ref }}$ to stabilize (17)-(18). Since $f_{t h}$ can only affect body $z$ direction, the UAV rotational dynamics will be coupled with the UAV translational dynamics by means of $\dot{\boldsymbol{w}}_{b}^{\text {ref }}$. The following theorem states the main result of the paper.

Theorem 2 (A stabilizing controller): Under the Assumption 1 and under the control law (10) with (15), define

$$
\begin{aligned}
f_{t h} & =-D_{z} \tilde{v}_{b, z}-g_{t, z}, \\
\dot{\boldsymbol{w}}_{b}^{\mathrm{ref}} & =-\boldsymbol{D}\left(\boldsymbol{D}_{w} \tilde{\boldsymbol{w}}_{b}-\overline{\boldsymbol{M}}_{t r}^{T} \tilde{\boldsymbol{v}}_{b}\right)+\frac{d}{d t}\left(\boldsymbol{Q} \boldsymbol{D}_{\phi} \boldsymbol{\phi}\right),
\end{aligned}
$$


where $\boldsymbol{D}, \boldsymbol{D}_{w}>0$ are diagonal control gain matrices, and $D_{z}>$ 0 is a scalar control gain. Moreover, $\tilde{\boldsymbol{w}}_{b}$ and $\tilde{\boldsymbol{v}}_{b}$ are

$$
\begin{gathered}
\tilde{\boldsymbol{w}}_{b}=\boldsymbol{w}_{b}+\boldsymbol{Q} \boldsymbol{D}_{\phi}\left(\boldsymbol{\phi}-\boldsymbol{\phi}^{\mathrm{des}}\right) \\
\tilde{\boldsymbol{v}}_{b}=\boldsymbol{v}_{b}+{ }^{b} \boldsymbol{R}_{g} \boldsymbol{D}_{r}\left(\boldsymbol{r}-\boldsymbol{r}^{\mathrm{des}}\right),
\end{gathered}
$$

where $\boldsymbol{D}_{r}, \boldsymbol{D}_{\phi}>0$ are diagonal gain matrices. Then the controlled UAV-M system is locally asymptotically stable if the gains $\boldsymbol{D}_{w}, \boldsymbol{D}_{\phi}$ are sufficiently large, and the control goal in Section III-A can be achieved.

Proof. See Section III-C.

The goal of the controller (19)-(20) is to achieve $\tilde{\boldsymbol{v}}_{b}$ and $\tilde{\boldsymbol{w}}_{b}=0$ which imply the first order stable error dynamics

$$
\boldsymbol{v}+\boldsymbol{D}_{r}\left(\boldsymbol{r}-\boldsymbol{r}^{\mathrm{des}}\right)=0 \text { and } \dot{\boldsymbol{\phi}}+\boldsymbol{D}_{\phi}\left(\boldsymbol{\phi}-\boldsymbol{\phi}^{\mathrm{des}}\right)=0
$$

because $\boldsymbol{v}_{b}={ }^{b} \boldsymbol{R}_{g} \boldsymbol{v}$ and $\boldsymbol{w}_{b}=\boldsymbol{Q} \dot{\boldsymbol{\phi}}$. Intuitively speaking, (23) is likely to be achievable because the closed-loop dynamics of (17) has a stable PD dynamics of $\tilde{\boldsymbol{v}}_{b}$, and that of (18) has a stable PD dynamics of $\tilde{\boldsymbol{w}}_{b}$. Note that the first 2 by 2 of $\overline{\boldsymbol{M}}_{t r} \boldsymbol{D} \overline{\boldsymbol{M}}_{t r}^{T}$ which is relevant to body $x, y$ linear velocity is always positive definite (recall Assumption 1), and body $z$ direction has a dedicated control $f_{t h}$. However, the coupling caused by $\boldsymbol{w}_{b}$ in (17) and $\boldsymbol{v}_{b}$ in (18) makes the formal stability proof difficult. This difficulty can be overcome by employing singular perturbation theory, as presented in next section.

\section{Theoretical Derivation (Proof of Theorem 2)}

Singular perturbation analysis (also known as two-time scale analysis) will be applied to (17)-(18) by letting $\boldsymbol{D}_{w}=\boldsymbol{D}_{\phi}=$ $\frac{1}{\epsilon} \boldsymbol{I}_{3 \times 3}$ and $\boldsymbol{D}=\boldsymbol{I}_{3 \times 3}$ for simplicity with some $\epsilon>0$. First, let us investigate (18) whose closed-loop dynamics is

$$
\dot{\tilde{\boldsymbol{w}}}_{b}+\frac{1}{\epsilon} \tilde{\boldsymbol{w}}_{b}=\overline{\boldsymbol{M}}_{t r}^{T} \tilde{\boldsymbol{v}}_{b} .
$$

Noting that $\tilde{\boldsymbol{w}}_{b}=\boldsymbol{w}_{b}-\boldsymbol{w}_{b}^{\mathrm{des}}=\boldsymbol{Q}\left[\dot{\boldsymbol{\phi}}+\frac{1}{\epsilon}\left(\boldsymbol{\phi}-\boldsymbol{\phi}^{\mathrm{des}}\right)\right]$, (24) becomes

$$
\begin{aligned}
\boldsymbol{Q} & {\left[\ddot{\boldsymbol{\phi}}+\frac{2}{\epsilon} \dot{\boldsymbol{\phi}}+\frac{1}{\epsilon^{2}}\left(\boldsymbol{\phi}-\boldsymbol{\phi}^{\mathrm{des}}\right)\right]+\dot{\boldsymbol{Q}}\left[\dot{\boldsymbol{\phi}}+\frac{1}{\epsilon}\left(\boldsymbol{\phi}-\boldsymbol{\phi}^{\mathrm{des}}\right)\right] } \\
& =\overline{\boldsymbol{M}}_{t r}^{T} \tilde{\boldsymbol{v}}_{b} .
\end{aligned}
$$

Let us now introduce a new time variable

$$
\sigma=\frac{1}{\epsilon} t
$$

which flows $\epsilon$ times faster than the real world time scale $t$. For this reason, $\sigma$ will be called the fast time scale. In addition to the fast time scale, the fast variable is defined by

$$
z=\frac{1}{\epsilon^{2}}\left(\phi-\phi^{\mathrm{des}}\right) .
$$

Then, (25) becomes, as $\epsilon \rightarrow 0$,

$$
\boldsymbol{Q}\left[\boldsymbol{z}^{\prime \prime}+2 \boldsymbol{z}^{\prime}+\boldsymbol{z}\right]=\overline{\boldsymbol{M}}_{t r}^{T} \tilde{\boldsymbol{v}}_{b},
$$

where $\boldsymbol{z}^{\prime}=\frac{d}{d t / \epsilon} \boldsymbol{z}=\frac{d}{d \sigma} \boldsymbol{z}$.

On the other hand, (17) can be expressed as

$$
\boldsymbol{v}_{b}^{\prime}=\mathbf{0}
$$

in the time scale $\sigma$. Namely, $\boldsymbol{v}_{b}$ can be considered as a frozen variable in (28), and consequently, $z$ converges to $\boldsymbol{z}=\boldsymbol{Q}^{-1} \overline{\boldsymbol{M}}_{t r}^{T} \tilde{\boldsymbol{v}}_{b}$ exponentially fast in the time scale $\sigma$.

Now in the time scale $t$, noting that, as $\epsilon \rightarrow 0, \boldsymbol{w}_{b}=$ $\boldsymbol{Q} \dot{\boldsymbol{\phi}}=\epsilon \boldsymbol{Q} \boldsymbol{z}^{\prime} \rightarrow \mathbf{0}, \boldsymbol{\phi}-\boldsymbol{\phi}^{\mathrm{des}}=\epsilon^{2} \boldsymbol{z} \rightarrow \mathbf{0}$, and $\dot{\boldsymbol{w}}_{b}^{\mathrm{ref}}=\boldsymbol{Q} \boldsymbol{z}-$ $\overline{\boldsymbol{M}}_{t r}^{T} \tilde{\boldsymbol{v}}_{b} \rightarrow \mathbf{0}$, (17) can be written as

$$
\underbrace{\left[\begin{array}{ccc}
m & 0 & 0 \\
0 & m & 0 \\
0 & 0 & m
\end{array}\right]}_{=\overline{\boldsymbol{M}}_{t t}} \underbrace{\left[\begin{array}{ccc}
\cos \left(\gamma^{\mathrm{des}}\right) & \sin \left(\gamma^{\mathrm{des}}\right) & 0 \\
-\sin \left(\gamma^{\mathrm{des}}\right) & \cos \left(\gamma^{\mathrm{des}}\right) & 0 \\
0 & 0 & 1
\end{array}\right]}_{=\left.{ }^{b} \boldsymbol{R}_{g}\right|_{\alpha=\beta=0}} \dot{\boldsymbol{v}}
$$

because $\dot{\boldsymbol{R}}=\mathbf{0}, \boldsymbol{w}_{b}=0$, and $\phi=\phi^{\mathrm{des}}(\alpha=\beta=0$ and $\gamma=$ $\left.\gamma^{\text {des }}\right)$. Here, the Property 1 introduced in Section II is used. Consequently, the exponential convergence of $r_{z} \rightarrow r_{z}^{\text {des }}$ can be shown easily from the third row of (30). We can conclude exponential stability of $\boldsymbol{r} \rightarrow \boldsymbol{r}^{\text {des }}$ from the following Lemma.

Lemma 1: Assume that $\phi \equiv \phi^{\text {des }}$ (hence, $\boldsymbol{w}_{b}=\dot{\boldsymbol{w}}_{b} \equiv \mathbf{0}$ follows) and $r_{z} \equiv r_{z}^{\mathrm{des}}$. This assumption implies $r_{x}=r_{x}^{\mathrm{des}}$ and $r_{y}=r_{y}^{\text {des }}$.

Proof: If $r_{x} \neq r_{x}^{\text {des }}$ or $r_{y} \neq r_{y}^{\text {des }}$, then $\dot{\boldsymbol{w}}_{b}$ is nonzero which results in the contradiction, because $\dot{\boldsymbol{w}}_{b, x y}^{\text {ref }}=$ $\left.\overline{\boldsymbol{M}}_{t r}^{T}\right|_{\text {first } 2 \text { by } 2} \tilde{\boldsymbol{v}}_{b, x y}$ is nonzero from the Assumption 1. Hence $r_{x}=r_{x}^{\text {des }}$ and $r_{y}=r_{y}^{\text {des }}$ must hold.

In singular perturbation literature, (28) is called boundary layer system, and (30) is called reduced system. So far, we have investigated an extreme case $\epsilon \rightarrow 0$. To conclude exponential stability for a more relaxed condition, the following theorem which is a well-known result from the singular perturbation theory [22] can be applied.

Theorem 3 (Stability using singular perturbation analysis): If the boundary layer system is exponentially stable and the reduced system is exponentially stable, then there exists $\epsilon^{*}$ such that the original system is exponentially stable for 0 $<\epsilon<\epsilon^{*}$.

Hence, according to the cascade structure (Theorem 1) and the singular perturbation analysis (Theorem 3), the controlled UAV-M system is asymptotically stable for small enough $\epsilon$ (or sufficiently large $\boldsymbol{D}_{w}$ and $\boldsymbol{D}_{\phi}$ ).

\section{DISCUSSION}

\section{A. Working Principle and Gain Selection Strategy}

The theoretical derivation of the proposed controller starts from Fig. 3 which states that the analysis of UAV dynamics can be performed without considering the manipulator dynamics explicitly (but, the static part should be still taken into account). Namely, having a reliable control scheme for the manipulator, the UAV control can be designed in the decoupled manner. Howevever, this does mean that the UAV and manipulator are controlled independently, because the feedback linearizing action (10) contains coupling.

Remark 1: A cascaded control structure is often understood as a cascade of fast and slow subsystems. However, Theorem 1 requires asymptotic stability of the subsystems, and does not ask about convergence speed. It is obvious that, under the proposed approach, the UAV cannot be asymptotically stabilized while the manipulator is moving. But, this does not mean that the UAV-M 


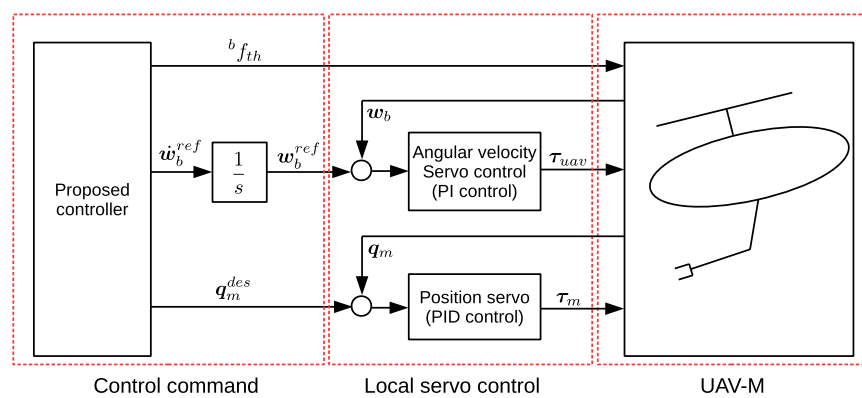

Fig. 4. Based on the working principle, the proposed controller can be applied not only to the torque controlled UAV-M, but also to the (partially) servo controlled UAV-M.
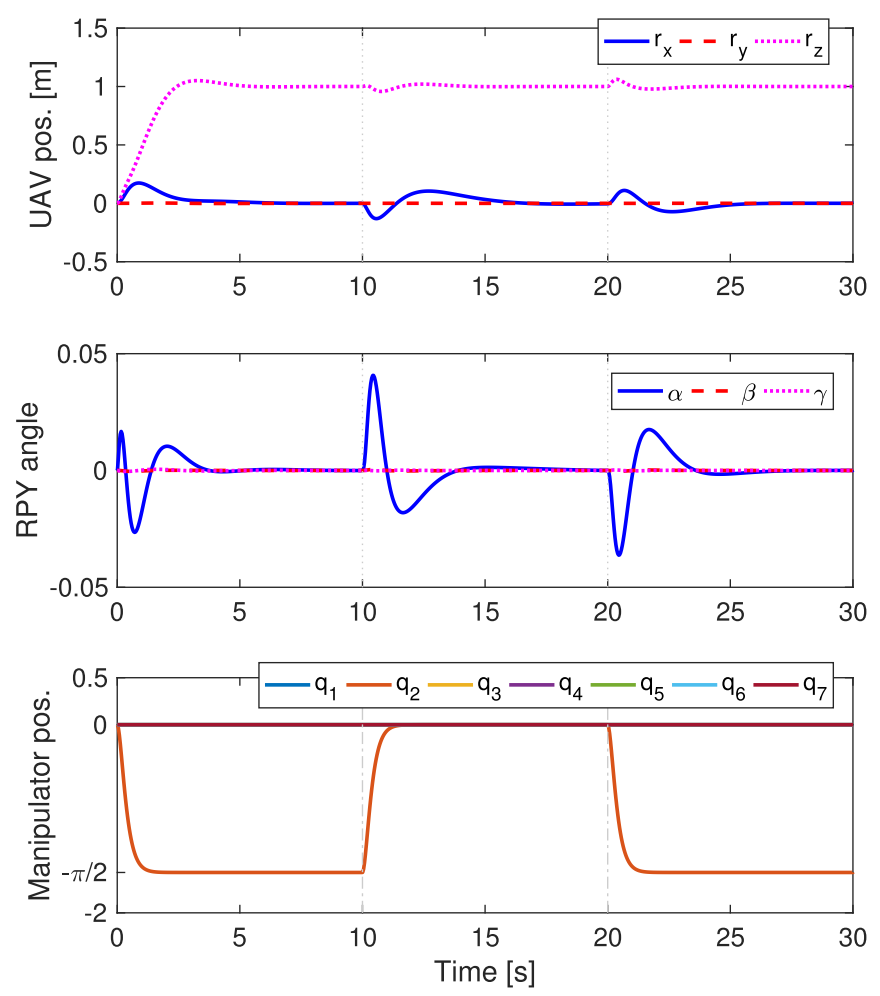

Fig. 5. The manipulator moved while hovering. Although the movement was close to the full swing, stability could be maintained.

will be destabilized. According to Theorem 2, the controlled UAV-M will be eventually asymptotically stabilized regardless of the manipulator's and the UAV's convergence speed.

The UAV control can be performed by $f_{t h}$ in (19) and $\dot{\boldsymbol{w}}_{b}^{\text {ref }}$ in (20). In principle, feedback linearization (10) should be applied to guarantee stability of which analysis requires perfect reference tracking. However, physically speaking, $\dot{\boldsymbol{w}}_{b}^{\text {ref }}$ design will be the most important part of the controller, and other control methods can be used as far as the reference tracking is reasonable. For example, local servo control can be applied instead of the feedback linearization, as will be further discussed in Section IV-B.

Because multiple control gains appear in the controller (19)(20), it is necessary to have a gain selection strategy. Roughly speaking, similar to (25), the closed-loop dynamics of (18) can be expressed as

$$
\begin{aligned}
& \boldsymbol{Q}\left[\ddot{\boldsymbol{\phi}}+\left(\boldsymbol{D}_{w}+\boldsymbol{D}\right) \dot{\boldsymbol{\phi}}+\boldsymbol{D} \boldsymbol{D}_{w} \boldsymbol{D}_{\phi}\left(\boldsymbol{\phi}-\boldsymbol{\phi}^{\mathrm{des}}\right)\right] \\
& =\boldsymbol{D} \overline{\boldsymbol{M}}_{t r}^{T b} \boldsymbol{R}_{g}\left(\boldsymbol{v}+\boldsymbol{D}_{r}\left(\boldsymbol{r}-\boldsymbol{r}^{\mathrm{des}}\right)\right) .
\end{aligned}
$$

If the right hand side is zero (meaning that there is no coupling between translational dynamics and rotational dynamics), $\phi$ will converge to the desired (recall that $\phi^{\text {des }}=\left[\begin{array}{lll}0 & 0 & \gamma^{\text {des }}\end{array}\right]^{T}$ ) regardless of the position error. In the proposed approach, position error exists on the right hand side to perturb the rotational dynamics when the asymptotic convergence is not achieved.

Therefore, the gains of left hand side determine the closedloop rotational dynamics, and those of the right hand side determine the amount of perturbation. Namely, if the left hand side gains are too large compared to the right hand side gains, the convergence speed will be very slow because the rotational dynamics excessively attenuates the perturbation. On the other hand, if the right hand side gains are too large compared to the left ones, the resulting behavior will be too shaky because of too large perturbation. The gain $D_{z}$ is rather independent to the other gains because $z$ direction is weakly coupled to the rotational dynamics (note that there is a dedicated control input, $\left.f_{t h}\right)$. Because the exponential convergence of $x, y$ directions is a consequence of the convergence of body $z$ direction (Lemma 1), $f_{\text {th }}$ should be designed to have strong control (see also Appendix).

Apart from $D_{z}$, we have to tune four gains $\boldsymbol{D}, \boldsymbol{D}_{w}, \boldsymbol{D}_{w}$, and $\boldsymbol{D}_{r}$ which are complicatedly coupled as shown in (31). In this letter, the gains were selected by the following procedure.

1) $\boldsymbol{D}$ is chosen based on $\bar{M}_{t r}^{T}$ which influences the right hand side. In particular, the first 2 by 2 block matrix of $\overline{\boldsymbol{M}}_{t r}^{T}$ (which is determined by the total mass and height of the CoM in the desired configuration) will be important, because it defines the coupling relation between roll, pitch and body $x, y$ directions.

2) The left hand side gains $\boldsymbol{D}_{w}$ and $\boldsymbol{D}_{\phi}$ are chosen to have (nearly) critical damped behavior of the rotational dynamics when the right hand side is zero.

3) $\boldsymbol{D}_{r}$ is chosen by increasing it from a small value. If the resulting response is not satisfactory, return to the second step with different $\boldsymbol{D}$ value.

As an example, we started with $\boldsymbol{D}=0.1 \boldsymbol{I}$ because

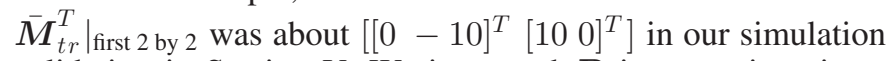
validation in Section V. We increased $\boldsymbol{D}$ in every iterations, and in each iterations, responses of $\boldsymbol{D}_{r}=0.1 \boldsymbol{I}, 0.5 \boldsymbol{I}$, and $\boldsymbol{I}$ were compared. After a few iterations, the following gains were selected and used in the implementation.

$$
\boldsymbol{D}_{w}=20 \boldsymbol{I}, \boldsymbol{D}_{\phi}=5 \boldsymbol{I}, \boldsymbol{D}=\boldsymbol{I}, \boldsymbol{D}_{r}=0.5 \boldsymbol{I} \text {, and } D_{z}=100 \text {. }
$$

The resulting behaviors for different $\boldsymbol{D}_{r}$ values will be shown in Section V-B to validate the discussion.

\section{B. Implementing Without Torque Servo Interface}

The resulting control law is given in $f_{t h}, \boldsymbol{\tau}_{\text {uav }}$, and $\boldsymbol{\tau}_{m}$. However, in practice, some systems including ours in Fig. 1(a), do not have torque servo interface. Our system, as an example, accepts $f_{\text {th }}$ command, but not $\boldsymbol{\tau}_{\text {uav }}$. Instead, we can command reference angular velocity to the UAV. For the manipulator, either position or torque can be commanded. 

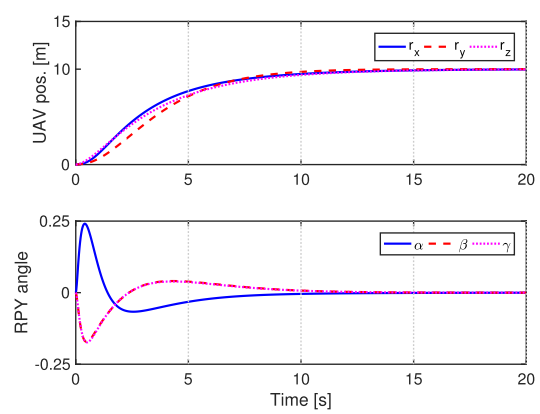

(a)
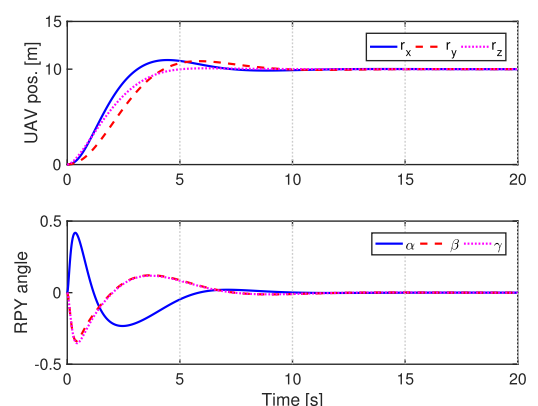

(b)
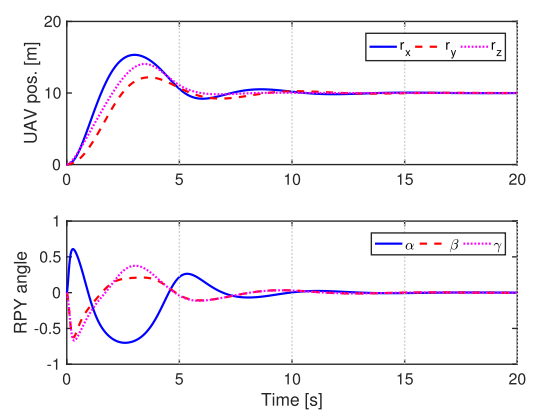

(c)

Fig. 6. Simulation results with increasing $\boldsymbol{D}_{r}$, while the other gains were fixed: Recall (32). The resulting motions were very similar to those of second order systems, as expected. The maximum overshoot of $r_{x}$ was $0 \rightarrow 2.6 \rightarrow 53.3 \%$. While the 10 to $90 \%$ rise time was improved with $\boldsymbol{D}_{r}$ ( $8.7 \rightarrow 2.0 \rightarrow 1.0$ sec

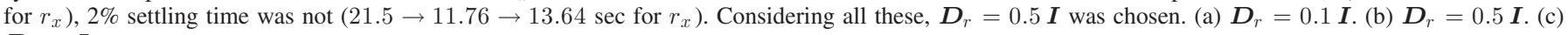
$\boldsymbol{D}_{r}=\boldsymbol{I}$.
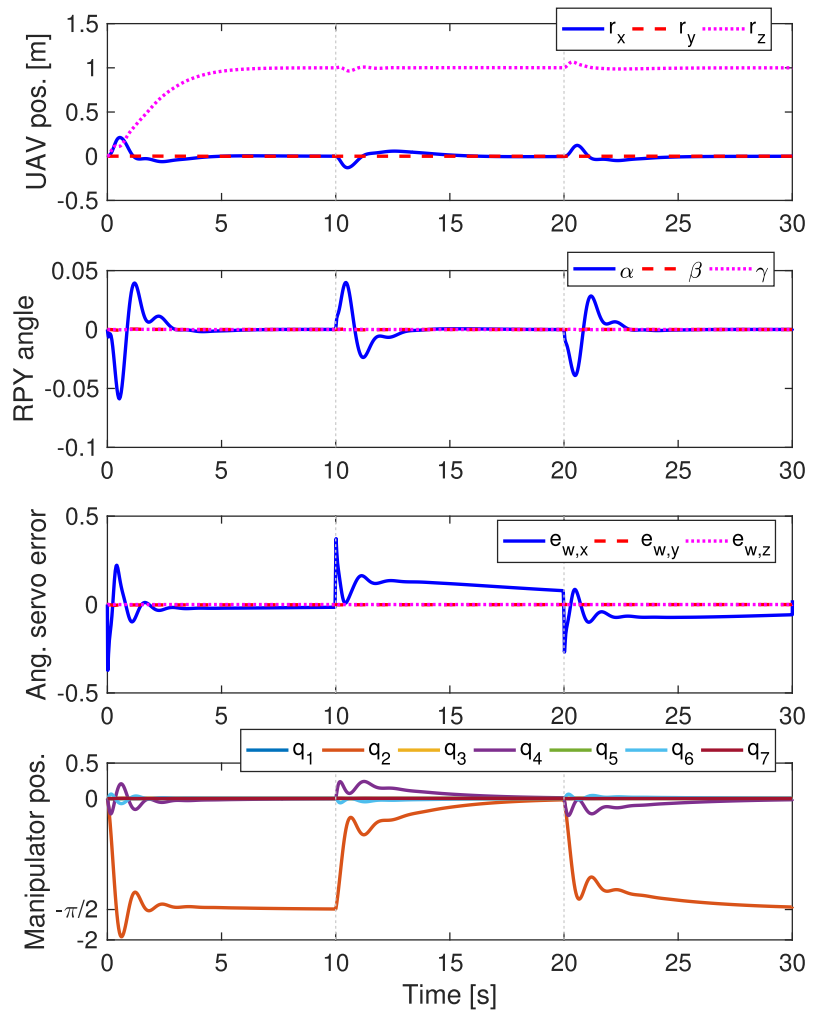

Fig. 7. The proposed controller was applied to UAV-M equipped with the position servo manipulator and angular velocity servo UAV, as shown in Fig. 4. Note that this implementation is almost model-free. $e_{w, x y z}$ in the third row denotes the tracking error of angular velocity. Despite imperfect reference tracking, stability was maintained.

Based on the working principle discussed in Section IV-A, the proposed controller can be applied to our system without any extensions, as shown in Fig. 4. Note that in this case, the controller is almost model-free: $\overline{\boldsymbol{M}}_{t r}^{T}$ is the only modeling term in $\dot{\boldsymbol{w}}_{b}^{\text {ref }}$ (see (20)). Fortunately, $\overline{\boldsymbol{M}}_{t r}=-\left.m^{b} \boldsymbol{r}_{C o M}^{\vee}\right|_{\boldsymbol{q}_{m}=\boldsymbol{q}_{m}^{\text {des }}}$ can be computed with a small effort.

\section{Limitation in Tracking Case}

This letter has tackled the regulation problem which is one of the most fundamental questions in control. In some real world scenarios, however, the robotic manipulator should be able to follow a certain trajectory. This section shows the limitation of the controller when applied to the tracking scenario. When the robotic manipulator is following a trajectory, say, $\boldsymbol{q}_{m}^{\mathrm{des}}(t), \dot{\boldsymbol{q}}_{m}^{\mathrm{des}}(t), \ddot{\boldsymbol{q}}_{m}^{\mathrm{des}}(t)$, the internal dynamics is

$$
\overline{\boldsymbol{M}}_{t t} \dot{\boldsymbol{v}}_{b}+\overline{\boldsymbol{M}}_{t r} \dot{\boldsymbol{w}}_{b}^{\mathrm{ref}}+\overline{\boldsymbol{M}}_{t m} \ddot{\boldsymbol{q}}_{m}^{\mathrm{des}}+\overline{\boldsymbol{C}}_{t} \boldsymbol{\xi}+\boldsymbol{g}_{t}=\left(\begin{array}{c}
0 \\
0 \\
f_{t h}
\end{array}\right) .
$$

Compared to (12), $\ddot{\boldsymbol{q}}_{m}^{\text {ref }}$ is replaced by $\ddot{\boldsymbol{q}}_{m}^{\text {des }}$. For the regulation case, once $\boldsymbol{q}_{m}$ has converged to the desired value $\boldsymbol{q}_{m}^{\text {des }}$, the influence of the manipulator dynamics on the internal dynamics (33) is gone. However, in the tracking case, even if the perfect tracking is achieved, the influence of the manipulator still exists because $\ddot{\boldsymbol{q}}_{m}^{\text {des }}$ (and $\dot{\boldsymbol{q}}_{m}^{\text {des }}$ ) is not zero. From (33), it is clear that the amount of manipulator's motion will directly impact the internal dynamics (i.e., the UAV translational dynamics). This feature will be further discussed in the simulation studies.

\section{Simulation VALIDATION}

To show that the proposed controller is indeed a stabilizing one, simulation studies are provided in this section. In addition, the discussions in Section IV are also validated. The rigid body dynamics (3) was used to simulate the UAV-M in Fig. 1. The UAV which has $37.6 \mathrm{~kg}$ mass with $\operatorname{diag}\{1.460 .361 .46\} \mathrm{kg} \cdot \mathrm{m}^{2}$ inertia, and 7 DoF DLR light weight robot (LWR) manipulator $(14 \mathrm{~kg})$ were used in the simulation. The LWR was mounted $32 \mathrm{~cm}$ away from the CoM of the UAV. The simulated UAV-M with $\boldsymbol{q}_{m}=\mathbf{0}$ is shown in Fig. 1(b).

\section{A. Validation of the Proposed Controller}

To validate the proposed controller, we performed the following task:

- UAV position: $\boldsymbol{r}^{\mathrm{des}}=\mathbf{0} \rightarrow\left[\begin{array}{lll}0 & 0 & 1\end{array}\right]^{T} \mathrm{~m}$.

- UAV orientation: $\phi^{\mathrm{des}}=\mathbf{0}$.

- manipulator position: $\boldsymbol{q}_{m}^{\text {des }}=\mathbf{0} \rightarrow\left[\begin{array}{lll}0 & -\frac{\pi}{2} & \mathbf{0}_{1 \times 5}\end{array}\right]^{T} \rightarrow \mathbf{0}$, periodically.

Here, the arrow $\rightarrow$ represents the step command. For the manipulator position, only the second joint was commanded to $-\pi / 2$ to generate nearly full swing motion that significantly influences the UAV dynamics. It is shown in [11] that, while 

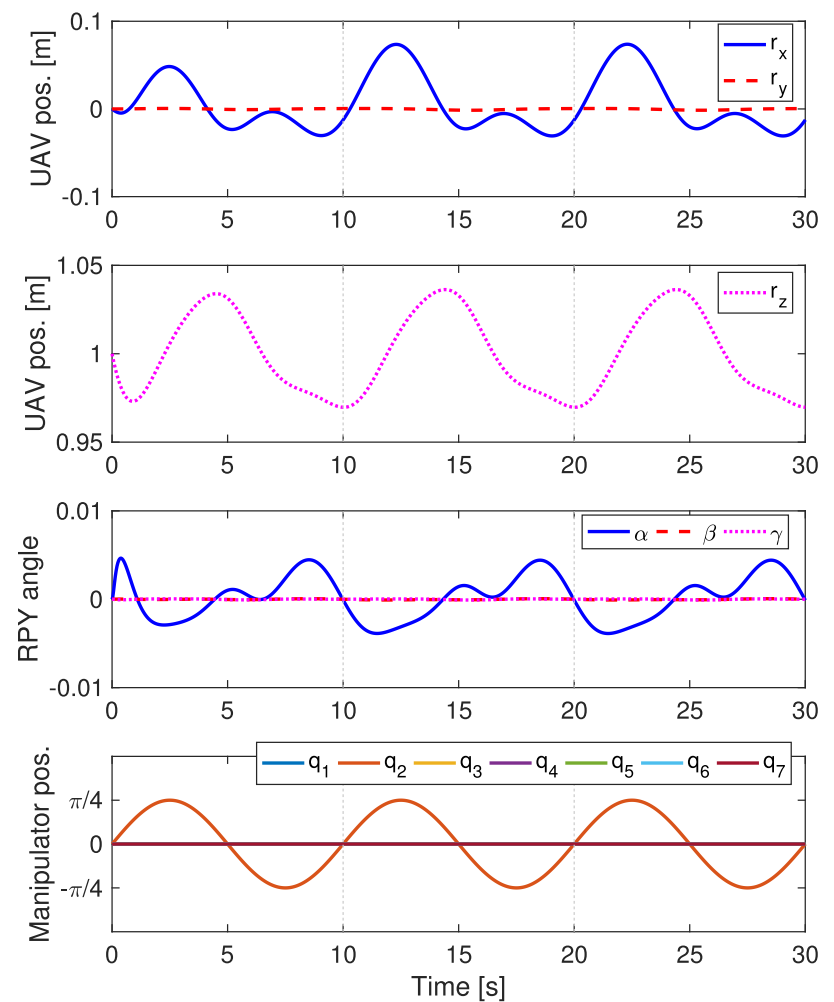

(a)
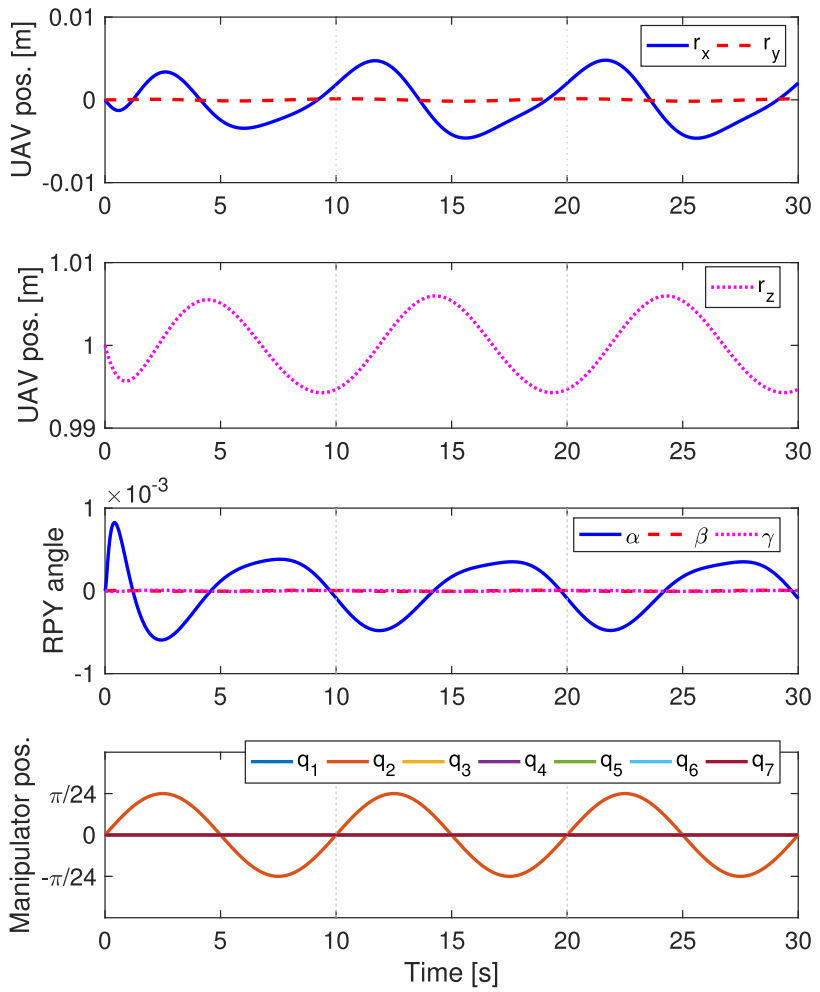

(b)

Fig. 8. Simulation results for the tracking case. Left: large and fast trajectory (the 2nd joint travels $90^{\circ}$ in the fully stretched configuration). Right: small and slow trajectory (the 2nd joint travels $15^{\circ}$ ). The maximum deviations of $r_{x}$ were about $0.073 \mathrm{~m}$ (left) and $0.0046 \mathrm{~m}$ (right).

hovering, the manipulator's simple swing can destabilize the UAV-M system.

It is clear that the UAV position will be influenced as the robotic manipulator moves due to the dynamic coupling (recall (33)). In the simulation result (Fig. 5), as soon as the position error occurred due to the manipulator's motion, angular motion was generated according to $\dot{\boldsymbol{w}}_{b}^{\text {ref }}$ in (20). As a result, asymptotic stability could be achieved. Note also that the $\boldsymbol{q}_{m}$ is not influenced by UAV motions, because the closed-loop manipulator dynamics (16) is decoupled via feedback linearization and pole-placement.

\section{B. Validation of the Gain Selection Strategy}

In this section, to validate the gain selection strategy discussed in Section IV-A, $\boldsymbol{D}_{r}$ was increased as $\boldsymbol{D}_{r}=0.1 \boldsymbol{I} \rightarrow 0.5 \boldsymbol{I} \rightarrow$ $I$, while the other gains were fixed according to (32).

- UAV position: $\boldsymbol{r}^{\mathrm{des}}=\mathbf{0} \rightarrow\left[\begin{array}{lll}10 & 10 & 10\end{array}\right]^{T} \mathrm{~m}$.

- UAV orientation: $\phi^{\mathrm{des}}=\mathbf{0}$.

- manipulator position: $\boldsymbol{q}_{m}^{\mathrm{des}}=\mathbf{0}$.

The results are summarized in Fig. 6. Although asymptotic stability could be achieved for every gains, more shaky motion was generated as the gain $\boldsymbol{D}_{r}$ increased (see the $y$ axis of RPY angle plots). Considering the resulting responses, $\boldsymbol{D}_{r}=0.5 \boldsymbol{I}$ was chosen as the final control gain.

\section{Implementation on Non-Torque Controllable UAV-M}

To validate the discussion in Section IV-B, the proposed controller was implemented to the UAV-M which is not equipped with a torque servo interface. The UAV angular velocity servo and manipulator position servo were implemented using local PI and PID control, as shown in Fig. 4. The same task with Section V-A was performed. Although the control performance was reduced because of the imperfect reference tracking, the controlled UAV-M was stable (Fig. 7).

\section{Tracking Control}

As discussed in Section IV-C, UAV positioning error will occur when the tracking control is applied. To investigate this in more detail, we compare two tasks:

- For both tasks, UAV position: hovering in $\boldsymbol{r}=\left[\begin{array}{lll}0 & 0 & 1\end{array}\right]^{T} \mathrm{~m}$.

- For both tasks, UAV orientation: $\phi^{\mathrm{des}}=\mathbf{0}$.

- For task 1, manipulator position: $\boldsymbol{q}_{m}^{\text {des }}(t)=\left[0 ; \frac{\pi}{4} \sin \right.$ $\left.\left(\frac{\pi}{10} t\right) \mathbf{0}_{1 \times 5}\right]^{T}$. For task $2: \quad \boldsymbol{q}_{m}^{\mathrm{des}}(t)=\left[0 ; \frac{\pi}{24} \sin \left(\frac{\pi}{10} t\right)\right.$ $\left.\mathbf{0}_{1 \times 5}\right]^{T}$.

The task 1 and 2 respectively imply $90^{\circ}$ and $15^{\circ}$ swing with 10 $\mathrm{sec}$ period in the fully stretched configuration. Since the poleplacement was applied to the manipulator, $\boldsymbol{q}_{m}$ followed the desired trajectory exactly. However, as discussed in Section IV-C, the amount of position error was directly affected by that of manipulators motion; the maximum deviation of $r_{x}$ was about $0.073 \mathrm{~m}$ for the task 1 , and was about $0.0046 \mathrm{~m}$ for the task 2 (see Fig. 8).

\section{CONCLUSION AND FUTURE WORKS}

In this letter, a stabilizing regulation controller for UAV-M systems is proposed. Although the regulation is a fundamental question in control problems, to the best of authors' knowledge, 
this is the first time showing the full proof. According to the working principle discussed in Section IV, the controllers for the UAV and the manipulator can be designed in the decoupled manner. Since multiple gains appear in the control law, a gain tuning strategy is discussed. In addition, the proposed approach can be applied to the UAV-M which has angular velocity servo instead of torque servo for UAV part. In this case, the implementation is almost model-free. Limitation on the tracking case is also discussed. These features were validated in the simulation studies.

Many unsolved problems are left. For example, the proposed approach should be extended to (i) tracking control, and (ii) Cartesian space control to tackle more practically relevant scenarios. Furthermore, (iii) to accomplish the manipulation tasks, interaction with the environment should be incorporated in the future.

\section{APPENDIX}

The output variable $\boldsymbol{y}$ can be selected alternatively by

$$
\begin{aligned}
\boldsymbol{y} & =\left[\begin{array}{l}
\mathbf{0}_{(4+n) \times 2} \\
\boldsymbol{I}_{(4+n) \times(4+n)}
\end{array}\right] \boldsymbol{\xi} \\
& =\left(\begin{array}{c}
v_{b, z} \\
\boldsymbol{w}_{b} \\
\dot{\boldsymbol{q}}_{m}
\end{array}\right)
\end{aligned}
$$

Now, the pole-placement technique can be further applied to $v_{b, z}$ and/or $\gamma$. But, still, $\boldsymbol{w}_{b, x y}$ must be coupled with $\boldsymbol{v}_{b, x y}$ to guarantee stability. It would be worthwhile to mention that, in the authors' experience, (34) showed better result in terms of control performance (e.g., convergence speed). This can be understood from the discussion in Section IV-A. However, (34) results in complicated model-based control law for $f_{t h}$, while (19) is model-free.

\section{REFERENCES}

[1] M. Orsag, C. Korpela, and P. Oh, "Modeling and control of MM-UAV: Mobile manipulating unmanned aerial vehicle," J. Intell. Robot. Syst., vol. 69 , pp. 227-240, 2013.

[2] A. Suarez, G. Heredia, and A. Ollero, "Lightweight compliant arm with compliant finger for aerial manipulation and inspection," in Proc. IEEE/RSJ Int. Conf. Intell. Robots Syst., 2016, pp. 4449-4454.

[3] B. Yüksel, N. Staub, and A. Franchi, "Aerial robots with rigid/elastic-joint arms: Single-joint controllability study and preliminary experiments," in Proc. IEEE/RSJ Int. Conf. Intell. Robots Syst., 2016, pp. 1667-1672.
[4] M. Ryll et al., "6D physical interaction with a fully actuated aerial robot," in Proc. IEEE Int. Conf. Robot. Autom., 2017, pp. 5190-5195.

[5] H. Yang and D. Lee, "Dynamics and control of quadrotor with robotic manipulator," in Proc. IEEE Int. Conf. Robot. Autom., 2014, pp. 5544 5549.

[6] G. Garofalo, B. Henze, J. Englsberger, and C. Ott, "On the inertially decoupled structure of the floating base robot dynamics," IFAC-PapersOnLine, vol. 48, no. 1, pp. 322-327, 2015.

[7] M. Karrer, M. Kamel, R. Siegwart, and M. Chli, "Real-time dense surface reconstruction for aerial manipulation," in Proc. IEEE/RSJ Int. Conf. Intell. Robots Syst., 2016, pp. 1601-1608.

[8] R. Rossi, A. Santamaria-Navarro, J. Andrade-Cetto, and P. Rocco, "Trajectory generation for unmanned aerial manipulators through quadratic programming," IEEE Robot. Autom. Lett., vol. 2, no. 2, pp. 389-396, Apr. 2017.

[9] A. Pumarola, A. Vakhitov, A. Agudo, A. Sanfeliu, and F. Moreno-Noguer, "PL-SLAM: Real-time monocular visual SLAM with points and lines," in Proc. IEEE Int. Conf. Robot. Autom., 2017, pp. 4503-4508.

[10] S. Rajappa, M. Ryll, H. H. Bülthoff, and A. Franchi, "Modeling, control and design optimization for a fully-actuated hexarotor aerial vehicle with tilted propellers," in Proc. 2015 IEEE Int. Conf. Robot. Autom., 2015, pp. 4006-4013.

[11] F. Huber et al., "First analysis and experiments in aerial manipulation using fully actuated redundant robot arm," in Proc. IEEE/RSJ Int. Conf. Intell. Robots Syst., 2013, pp. 3452-3457.

[12] K. Kondak et al., "Aerial manipulation robot composed of an autonomous helicopter and a 7 degrees of freedom industrial manipulator," in Proc. IEEE Int. Conf. Robot. Autom., 2014, pp. 2107-2112.

[13] M. Laiacker, F. Huber, and K. Kondak, "High accuracy visual servoing for aerial manipulation using a 7 degrees of freedom industrial manipulator," in Proc. IEEE/RSJ Int. Conf. Intell. Robots Syst., 2016, pp. 1631-1636.

[14] C. Korpela, M. Orsag, M. Pekala, and P. Oh, "Dynamic stability of a mobile manipulating unmanned aerial vehicle," in Proc. IEEE Int. Conf. Robot. Autom., 2013, pp. 4922-4927.

[15] M. Orsag, C. Korpela, S. Bogdan, and P. Oh, "Dexterous aerial robotsmobile manipulation using unmanned aerial systems," IEEE Trans. Robot., vol. 33, no. 6, pp. 1453-1466, Dec. 2017.

[16] Y. Yu, X. Ding, and J. J. Zhu, "Dynamic modeling and control for aerial arm-operating of a multi-propeller multifunction aerial robot," $A d v$. Robot., vol. 31, pp. 665-679, 2017.

[17] J. Acosta, M. Sanchez, and A. Ollero, "Robust control of underactuated aerial manipulators via IDA-PBC," in Proc. 2014 IEEE 53rd Annu. Conf. Decis. Control, 2014, pp. 673-678.

[18] V. Lippiello and F. Ruggiero, "Cartesian impedance control of a UAV with a robotic arm,” IFAC Proc. Vol., vol. 45, no. 22, pp. 704-709, 2012.

[19] S. Kim, S. Choi, and H. J. Kim, "Aerial manipulation using a quadrotor with a two DOF robotic arm," in Proc. IEEE/RSJ Int. Conf. Intell. Robots Syst., 2013, pp. 4990-4995.

[20] M. Tognon, B. Yüksel, G. Buondonno, and A. Franchi, "Dynamic decentralized control for protocentric aerial manipulators," in Proc. IEEE Int. Conf. Robot. Autom., 2017, pp. 6375-6380.

[21] P. Seibert and R. Suarez, "Global stabilization of nonlinear cascade systems," Syst. Control Lett., vol. 14, no. 4, pp. 347-352, 1990.

[22] H. K. Khalil and J. Grizzle, Nonlinear Systems, vol. 3. Upper Saddle River, NJ, USA: Prentice-Hall, 2002. 